\title{
Regulation of Oxidative Stress in Horticultural Crops
}

\author{
Albert C. Purvis \\ Department of Horticulture, Coastal Plain Experiment Station, Tifton, GA 31793-0748
}

Refrigeration slows respiration and other metabolic processes that lead to ripening and senescence of fruits and vegetables. Thus in order to extend their shelf life, most fruits and vegetables are cooled as rapidly as possible after harvest and stored at low temperatures until they are consumed. However, the shelf life of many fruits and vegetables, especially those of tropical and subtropical origins, as well as some of temperate origin, is actually shortened when they are stored at temperatures below a certain threshold that is characteristic for a particular commodity. Injury resulting from storage at low but above freezing temperatures is termed chilling injury. It is becoming widely accepted that symptoms developing during and after chilling and other environmental stresses are actually a consequence of oxidative stress in the tissues (Scandalios, 1993). This presentation will focus on how abiotic (environmental) and biotic stresses imposed on plant tissues result in oxidative stress and how plant tissues regulate oxidative stress. It will be limited, however, to the production of active oxygen species (AOS) by the mitochondrial electron transport chain and describes two mechanisms present in plant mitochondria which limit their production.

Oxygen, the second most abundant component in the earth's atmosphere comprising almost $21 \%$ of the atmospheric gases, is essential for respiration and energy metabolism. Paradoxically, oxygen also oxidizes cellular constituents, ultimately resulting in loss of function of organelles and eventual cell death. It is interesting to note, however, that molecular diatomic oxygen usually does not react directly with organic molecules because its two unpaired electrons have parallel spins. Thus, oxygen has to be activated to react with organic molecules with electrons having antiparallel spins. Activation of oxygen requires the absorption of sufficient energy to reverse the spin of one of its unpaired electrons (forming singlet oxygen), or the stepwise monovalent reductions to superoxide, hydrogen peroxide, and hydroxyl radical. Reduction of oxygen to unreactive water requires four electrons.

Most ( $85 \%$ to $95 \%$ ) of the oxygen consumed by plant (and animal) tissues is reduced to water by the terminal oxidase(s) of the respiratory electron transport chain in the mitochondria. The remaining 5\% to $15 \%$ is either partially or fully reduced or incorporated into organic molecules by various other oxidases and oxygenases. Leakage of electrons from single-electron-reduced components of electron transfer systems results in the monovalent reduction of oxygen to superoxide which is rapidly dismutated (either spontaneously or enzymatically) to hydrogen peroxide. Hydrogen peroxide is relatively stable and can penetrate organellar membranes, but in the presence of ferrous iron it is further reduced to the highly reactive and detrimental hydroxyl radical. It is estimated that $2 \%$ to $4 \%$ of the oxygen consumed during normal metabolism is monovalently reduced to superoxide (Liu and Huang, 1996). Superoxide and hydrogen peroxide production increases in stressed plant tissues.

Superoxide and hydrogen peroxide have important useful functions in several cellular processes, such as cell wall lignification, detoxification of xenobiotics, and the hypersensitive response to pathogens (McKersie and Leshem, 1994). They also serve as signals or messengers to trigger an increase in the production of antioxidants and active oxygen scavenging enzymes (Vranová et al., 2002) and are involved in programmed cell death (Fath et al., 2002) and processes related to the senescence of plant tissues and fruit ripening (Dhindsa et al., 1981). However, AOS, especially the hydroxyl radical, also damage plant cells by peroxidizing membrane lipids and proteins and inducing lesions in DNA that result in lethal deletions and mutations (McKersie and Leshem, 1994).

The level of AOS in plant tissues is regulated, in part, by the elaborate scavenging system that includes the active oxygen scavenging enzymes

Received for publication 2 Feb. 2004. Accepted for publication 2 Feb. 2004. Corresponding author; e-mail purvis@tifton.uga.edu. (superoxide dismutase, catalase and the various peroxidases) and the lipid-soluble ( $\alpha$-tocopherol and the carotenoids) and water-soluble (ascorbic acid, glutathione and the flavonoids) antioxidants (Noctor and Foyer, 1998). The manifestation of injury of plant tissues subjected to temperature (low and high), drought, and other environmental stresses results when production and scavenging of AOS by antioxidants and active oxygen scavenging enzymes are compromised.

Since mitochondria are the major consumers of oxygen, they are also a major site of production of AOS (Purvis, 2003). The respiratory electron transport chain, located in the inner membrane of the mitochondria (of both plant and animal cells), is made up of five multiprotein complexes and a dynamic ubiquinone pool which is present in large (10-fold) molar excess of other components of the electron transport chain (Fig. 1). The ubiquinone molecule consists of a quinone synthesized from the shikimic acid pathway and a prenyl tail composed of six to 11 isoprenoid units derived from the mevalonic acid pathway or the glyceraldehyde/pyruvate (Rohmer) pathway (Disch et al., 1998). The hydrophobic prenyl tail groups are closely associated with the lipid molecules in the inner membrane. Unlike most other membranes, the mitochondrial inner membrane has a high (about 4:1) protein to lipid ratio. The hydrophobic quinone head groups are alternately reduced and oxidized and serve as a sink for respiratory electrons from complexes I and II (NADH and succinate dehydrogenases, respectively). In addition to complexes I and II, plant mitochondria have a calcium-activated NADH dehydrogenase located on the exterior surface of the inner membrane and a rotenone-insensitive NADH dehydrogenase (complex I activity is inhibited with rotenone) located on the matrix side of the inner membrane that also reduce the ubiquinone pool (Fig. 1). Thus, each ubiquinone molecule can be reduced to ubiquinol by two electrons derived from respiratory substrates oxidized by either the TCA cycle enzymes in the mitochondrial matrix or the dehydrogenases located in the cytoplasm. Ubiquinol is subsequently oxidized by complex III (the cytochrome $b c_{1}$ complex) with the transfer of electrons one at a time through the cytochrome path to complex IV (cytochrome oxidase) which ultimately reduces oxygen to water by the simultaneous transfer of four electrons. A relatively high proportion (almost half) of the ubquinone, however, does not appear to be reduced/oxidized by the respiratory electron transport chain (Wagner and Wagner, 1995) and may participate in the structure of the inner membrane or serve as an antioxidant (Beyer, 1992).

Electron flow through three of the protein complexes (complexes I, III, and IV) develops a proton electrochemical gradient across the inner membrane (Fig. 1). This electrochemical gradient is dissipated, i.e., protons flow back across the mitochondrial inner membrane into the matrix, during the phosphorylation of ADP to ATP by complex V (ATP synthase). Thus, the continuous flow of electrons through the electron transport chain to oxygen requires rapid turnover of ATP to provide ADP. The external calcium-activated NADH dehydrogenase and the internal rotenone-insensitive NADH dehydrogenase, neither of which develops a proton electrochemical gradient across the inner membrane (Fig. 1), therefore may become more active in stressed plant tissues when the turnover of ATP is slow (Møller, 2001). Restriction or disruption of electron flow through the electron transport chain, such as occurs during resting (state 4) respiration (i.e., when all available ADP has been phosphorylated to ATP) and in stressed plant tissues, results in a substantial decrease in oxygen consumption by cytochrome oxidase. In consequence, a high concentration of oxygen builds up in the mitochondria and some of the components of the electron transport chain remain reduced. The high intra-mitochondrial oxygen concentration and high redox status of the electron transport chain favor leakage of electrons from single-electron-reduced components to molecular oxygen, resulting in increased production of AOS (Skulachev, 1997). Two sites in the mitochondrial electron transport chain where single electrons can leak to molecular oxygen reducing it to superoxide 


\section{Intermembrane Space}

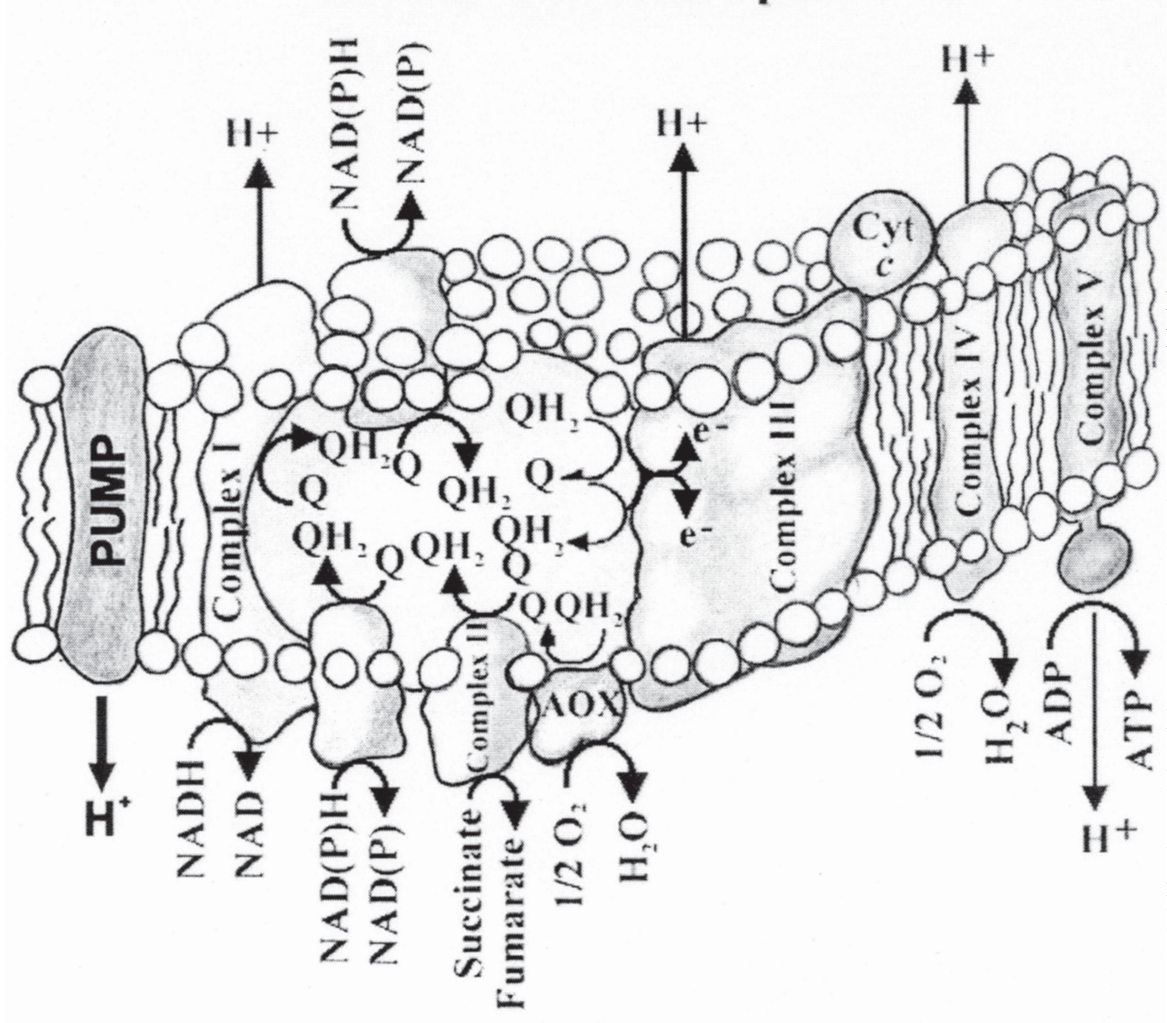

\section{Matrix}

are the flavoprotein components of the dehydrogenases, especially complex I, and the cytochrome $b c_{1}$ complex (complex III) (Rich and Bonner, 1978).

An obvious way to reduce the production of AOS in the tissues of fruits and vegetables is to store them in low oxygen concentrations (which forms a part of the basis of controlled atmosphere storage). Indeed, superoxide production increases when the oxygen concentration in the mitochondrial mileau increases (Boveris and Chance, 1973). However, there are also mechanisms in plant mitochondria that can regulate their production of AOS. Plant mitochondria have two ways to relieve the restriction of electron flow through the electron transport chain without producing AOS. The plant uncoupling mitochondrial protein (PUMP) discovered by Vercesi et al. (1995) dissipates the proton electrochemical gradient across the mitochondrial inner membrane without the production of ATP (Fig. 1). It is similar to the uncoupling mitochondrial protein of mammalian brown adipose tissue required for nonshivering thermogenesis in newborn, cold-acclimated and hibernating mammals (Tyler, 1992). PUMP is activated by free fatty acids, especially linoleic acid, and inhibited by purine nucleotides (e.g., GTP). Protonated fatty acids readily cross the mitochondrial inner membrane and the anionic fatty acids are removed by the PUMP from the matrix back into the intermembrane space resulting in fatty acid recycling and mitochondrial uncoupling (Jezek et al., 1996). In consequence, mitochondrial resting potential (state 4) respiration increases with a concomitant decrease in membrane electrochemical potential.

In addition to cytochrome oxidase, plant mitochondria have an alternative oxidase (AOX) located on the matrix side of the inner membrane, that oxidizes ubiquinol directly without producing a proton electrochemical gradient across the inner membrane (Fig. 1). Similar to cytochrome oxidase, the alternative oxidase reduces oxygen directly to water with four electrons. Since electron flow through the alternative oxidase is not coupled with the synthesis of ATP, alternative oxidase activity can reduce the redox potential of the components of the electron transport chain and at the same time lower the oxygen concentration in the mitochondria, thus reducing the potential for leakage of electrons
Fig. 1. A three-dimensional view of the respiratory electron transport chain in the mitochondrial inner membrane from a plant cell. The five multi-protein complexes are complex I (rotenone-sensitive NADH dehydrogenase), complex II (succinate dehydrogenase), complex III (the cytochrome $b c_{1}$ complex), complex IV (cytochrome oxidase), and complex V (ATP synthase). The mobile, dynamic ubiquinone pool is present in large molar excess to other components. Additional components in plant mitochondria include a calcium-activated external NADH dehydrogenase, a rotenone-insensitive internal NADH dehydrogenase, a plant uncoupling mitochondrial protein (PUMP) and an alternative oxidase (AOX).

from single-electron-reduced components to oxygen (Purvis and Shewfelt, 1993). Although the alternative oxidase is ubiquitous in plant mitochondria, it is inducible in most plant tissues by several stresses, including wounding, low temperature, osmotic, and drought (Wagner and Krab, 1995). Maximal activity requires reduction of the sulfhydryl groups on the enzyme and the presence of an $\alpha$-keto acid, such as pyruvate, the end product of glycolysis (Umbach et al., 1994). Pyruvate interacts directly with sulfhydryl groups on the enzyme (Umbach et al., 1994) and lowers the reduced ubiquinone to total ubiquinone ratio required for maximal activity (Day et al., 1995). Recent studies with transgenic cultured tobacco cells overexpressing and underexpressing the alternative oxidase show clearly that alternative oxidase activity lowers the production of AOS when respiratory electron flow is restricted with cytochrome pathway respiratory inhibitors (Maxwell et al., 1999) and during phosphate-limited growth (Parsons et al., 1999). Thus, the alternative oxidase dissipates redox energy without building up the phosphate potential of the mitochondria.

In summary, the injuries sustained by sensitive plant tissues exposed to environmental (abiotic) and biotic stresses are actually a consequence of oxidative stress. The enhanced respiration experienced both during and after stress can overload the reducing power and phosphate potential of the mitochondria, resulting in increased leakage of electrons from the single-electron-reduced components of the electron transport chain directly to molecular oxygen and an increased production of AOS. Plant mitochondria have two energy-dissipating systems, which can work as safety valves when this occurs. The PUMP consumes the proton electrochemical gradient by recycling fatty acids across the mitochondrial inner membrane thus diverting the energy from oxidative phosphorylation. The alternative oxidase, which is not controlled by the energy status of the cell, directly decreases the reducing power of the electron transport chain in the mitochondrial inner membrane. The PUMP and the alternative oxidase are probably not active in the same plant tissue simultaneously. (Holtzapffel et al., 2002). Obviously, the mitochondrial respiratory electron transport chain is only one of several sites in plant cells where AOS are produced. Other sites of AOS production include the chloroplasts, endoplasmic reticulum, peroxisomes, glyoxysomes, plasma membranes and cell walls. These sites are especially important in wound healing, cell wall lignification and the hypersensitive response to pathogens and insects. Thus, antioxidants and active oxygen scavenging enzymes are still important in controlling the overall level of AOS in plant cells.

\section{Literature Cited}

Beyer, R.E. 1992. An analysis of the role of coenzyme Q in free radical generation and as an antioxidant. Biochem. Cell Biol. 70:390-403.

Boveris, A. and B. Chance. 1973. The mitochondrial generation of hydrogen peroxide. Biochem. J. 134:707-716.

Day, D.A., J. Whelan, A.H. Millar, J.N. Siedow, and J.T. Wiskich. 1995. Regulation of the alternative oxidase in plants and fungi. Austral. J. Plant Physiol. 22:497-509. 
Dhindsa, R.S., P. Plumb-Dhindsa, and T.A. Thorpe. 1981. Leaf senescence: Correlated with increased levels of membrane permeability and lipid peroxidation, and decreased levels of superoxide dismutase and catalase. J. Expt. Bot. 32:93-101.

Disch, A., A. Hemmerlin, T.J. Bach, and M. Rohmer. 1998. Mevalonate-derived isopentenyl diphosphate is the biosynthetic precursor of ubiquinone prenyl side chain in tobacco BY-2 cells. Biochem. J. 331:615-621.

Fath, A., P. Bethke, V. Beligni, and R. Jones. 2002. Active oxygen and cell death in cereal aleurone cells. J. Expt. Bot. 53:1273-1282.

Holtzapffel, R.C., P.M. Finnegan, A.H. Millar, M.R. Badger, and D.A. Day. 2002. Mitochondrial protein expression in tomato fruit during on-vine ripening and cold storage. Funct. Plant Biol. 29:827-834.

Jezek, P., A.D.T. Costa, and A.E. Vercesi. 1996. Evidence for anion-translocating plant uncoupling mitochondrial protein in potato mitochondria. J. Biol. Chem. 271:32743-32748.

Liu, S-s. and J-p. Huang. 1996. Coexistence of a "reactive oxygen cycle" with the "Q-cycle" in the respiratory chain-A hypothesis for generating, partitioning, and functioning of superoxide in mitochondria, p. 513-529. In: D. Moores (ed.). Natural antioxidants: Molecular mechanisms and health effects. AOCS Press, Champaign, Ill.

Maxwell, D.P., Y. Wang, and L. McIntosh. 1999. The alternative oxidase lowers mitochondrial reactive oxygen production in plant cells. Proc. Natl. Acad. Sci. USA 99:8271-8276.

McKersie, B.D. and Y.Y. Leshem. 1994. Oxidative stress, p. 15-54. In: B.D. McKersie and Y.Y. Leshem (eds.). Stress and stress coping in cultivated plants. Kluwer Academic Publ., Dordrecht, The Netherlands.

Møller, I. M. 2001. Plant mitochondria and oxidative stress: Electron transport, NADPH turnover, and metabolism of reactive oxygen species. Annu. Rev. Plant Physiol. Plant Mol. Biol. 52:561-591.
Noctor, G. and C.H. Foyer. 1998. Ascorbate and glutathione: Keeping active oxygen under control. Annu. Rev. Plant Physiol. Plant Mol. Biol. 49:249-279.

Parsons, H.L., J.Y.H. Yip, and G.C. Vanlerberghe. 1999. Increased respiratory restriction during phosphate-limited growth in transgenic tobacco cells lacking alternative oxidase. Plant Physiol. 121:1309-1320.

Purvis, A.C. 2003. How respiring plant cells limit the production of active oxygen species, p. 151-164. In: D.M. Hodges (ed.). Postharvest oxidative stress in horticultural crops. Food Products Press, New York.

Purvis, A.C. and R.L. Shewfelt. 1993. Does the alternative pathway ameliorate chilling injury in sensitive plant tissues? Physiol. Plant. 88:712-718.

Rich, P. R. and W.D. Bonner, Jr. 1978. The sites of superoxide anion generation in higher plant mitochondria. Arch. Biochem. Biophys. 188:206-213.

Scandalios, J.G. 1993. Oxygen stress and superoxide dismutases. Plant Physiol. 101:7-12.

Skulachev, V.P. 1997. Membrane-linked systems preventing superoxide formation. Biosci. Rpt. 17:347-366.

Tyler, D.D. 1992. The mitochondrion in health and disease. VCH Publ., Inc., New York.

Umbach, A.L., J.T. Wiskich, and J.N. Siedow. 1994. Regulation of alternative oxidase kinetics by pyruvate and intermolecular disulfide bond redox status in soybean seedling mitochondria. FEBS Lett. 348:181-184.

Vercesi, A.E., I.S. Martins, M.A.P. Silva, and H.M.F. Lelte. 1995. PUMPing plants. Nature 375:24

Vranová, E., D. Inzé, and F. Van Breusegem. 2002. Signal transduction during oxidative stress. J. Expt. Bot. 53:1227-1236.

Wagner, A.M. and K. Krab. 1995. The alternative respiration pathway in plants. Role and regulation. Physiol. Plant. 95:318-325.

Wagner, A.M. and M.J. Wagner. 1995. Measurements of in vivo ubiquinone reduction levels in plant cells. Plant Physiol. 108:277-283. 